\title{
Paternal obesity is associated with IGF2 hypomethylation in newborns: results from a Newborn Epigenetics Study (NEST) cohort
}

\author{
Adelheid Soubry ${ }^{1 *}$, Joellen M Schildkraut ${ }^{1,2}$, Amy Murtha $^{3}$, Frances Wang ${ }^{1}$, Zhiqing Huang ${ }^{4}$, Autumn Bernal ${ }^{5}$,
} Joanne Kurtzberg ${ }^{6}$, Randy L Jirtle ${ }^{5}$, Susan K Murphy ${ }^{4}$ and Cathrine Hoyo ${ }^{7}$

See related commentary article here http://www.biomedcentral.com/1741-7015/11/30

\begin{abstract}
Background: Data from epidemiological and animal model studies suggest that nutrition during pregnancy may affect the health status of subsequent generations. These transgenerational effects are now being explained by disruptions at the level of the epigenetic machinery. Besides in vitro environmental exposures, the possible impact on the reprogramming of methylation profiles at imprinted genes at a much earlier time point, such as during spermatogenesis or oogenesis, has not previously been considered. In this study, our aim was to determine associations between preconceptional obesity and DNA methylation profiles in the offspring, particularly at the differentially methylated regions (DMRs) of the imprinted Insulin-like Growth Factor 2 (IGF2) gene.

Methods: We examined DNA from umbilical cord blood leukocytes from 79 newborns, born between July 2005 and November 2006 at Duke University Hospital, Durham, NC. Their mothers participated in the Newborn Epigenetics Study (NEST) during pregnancy. Parental characteristics were obtained via standardized questionnaires and medical records. DNA methylation patterns at two DMRs were analyzed by bisulfite pyrosequencing; one DMR upstream of IGF2 (IGF2 DMR), and one DMR upstream of the neighboring H19 gene (H19 DMR). Multiple regression models were used to determine potential associations between the offspring's DNA methylation patterns and parental obesity before conception. Obesity was defined as body mass index (BMI) $\geq 30 \mathrm{~kg} / \mathrm{m}^{2}$.

Results: Hypomethylation at the IGF2 DMR was associated with paternal obesity. Even after adjusting for several maternal and newborn characteristics, we observed a persistent inverse association between DNA methylation in the offspring and paternal obesity ( $\beta$-coefficient was $-5.28, P=0.003$ ). At the H19 DMR, no significant associations were detected between methylation patterns and paternal obesity. Our data suggest an increase in DNA methylation at the IGF2 and H19 DMRs among newborns from obese mothers, but a larger study is warranted to further explore the potential effects of maternal obesity or lifestyle on the offspring's epigenome.

Conclusions: While our small sample size is limited, our data indicate a preconceptional impact of paternal obesity on the reprogramming of imprint marks during spermatogenesis. Given the biological importance of imprinting fidelity, our study provides evidence for transgenerational effects of paternal obesity that may influence the offspring's future health status.
\end{abstract}

Keywords: Epigenetics, DNA methylation, IGF2, obesity, offspring, Newborn Epigenetics Study, Epidemiology

\footnotetext{
* Correspondence: adelheid.soubry@hotmail.com

'Duke Cancer Institute, Duke University Medical Center 2715, Durham, NC

27710, USA

Full list of author information is available at the end of the article
} 


\section{Background}

Environmental exposures acquired early in life have been correlated with persistent modifications of the epigenome. The epigenetic information in human cells is stored via mitotically heritable DNA methylation, organization of the chromatin structure (for example, histone modification), and regulatory RNAs. Together, these mechanisms are responsible for regulating gene expression during cellular differentiation during embryonic development and throughout life [1]. Our study focuses on the DNA methylation patterns of the imprinted Insulin-Like Growth Factor 2 (IGF2) gene, coding a well-characterized growth factor active throughout embryogenesis and fetal growth $[2,3]$. In normal human tissues only the paternal IGF2 allele is transcribed; and its imprinting is regulated by at least two differentially methylated regions (DMRs): one is located upstream of the three IGF2 promoters that are subject to imprinting (IGF2 DMR), and the other is located upstream of the neighboring non-coding H19 gene (H19 DMR). The latter region is part of the imprinting control region (ICR) which harbors binding sites for the zinc finger protein CTCF. During early development, imprint marks are erased in the primordial germ cells and new methylation imprints are established according to the germ cell. This has particularly been demonstrated at the IGF2 DMR [4] and H19 DMR [4,5] in spermatogenic human cell stages. The progressive imprint re-establishment of the DNA methylation imprint marks throughout human spermatogenesis leads to fully methylated IGF2 and H19 DMRs. Consequently, methylation is only present on the paternally inherited allele in the offspring. Shifts in methylation established at these DMRs can lead to loss of imprinting and transcription of IGF2 may be altered [6,7]. Hence, normal physiological mechanisms or homeostasis in the body may be skewed and lead to chronic diseases later in life. Until now, epidemiological studies have focused on maternal factors and especially in utero exposures to certain nutritional or environmental conditions as the potential explanation for such disruptions or shifts in methylation at the DMRs [8-10]. This can potentially contribute to a higher risk for obesity [11], chronic diseases at later age [12], including diabetes or cardiovascular diseases [13-15], or even cancer $[16,17]$. Animal experiments show that modification of maternal diet during development can influence metabolism in adulthood [18]. Although the underlying mechanism and crucial time points of exposure are not clear, changes in epigenetic regulation are now regarded as a highly plausible explanation for linking the associations between dietary exposures in early life to the onset of chronic diseases during adulthood. Several lines of evidence suggest that pre- or periconceptional obesity of the mother may affect metabolic programming [19-21].
Although paternal obesity is equally prevalent, the majority of the epidemiological studies to date suggest in utero exposures as the only possible origin of potential epigenomic modifications at birth. Obesity is associated with over-nutrition, unbalanced food intake (such as low vegetable consumption), and a sedentary lifestyle [22]. Consequently, elucidating the epigenetic risks associated with the current "Western" lifestyle on the next generations is crucial. In the current report we determined whether preconceptional obesity, in the mother or the father, is associated with methylation patterns at the IGF2 DMRs in newborns using DNA from leukocytes isolated from umbilical cord blood at birth. By including paternal obesity in our study we were able to examine a potential preconceptional impact of the environment on imprint mark reprogramming during male gametogenesis. Consequently, we found that paternal obesity is associated with a decrease in DNA methylation at the IGF2 DMR.

\section{Methods}

\section{Study participants}

We studied a subgroup of the first 98 families enrolled in the Newborn Epigenetics Study cohort. Seventy-eight mothers provided detailed information about the biological fathers. One mother gave birth to twins, bringing the total number of newborns with corresponding paternal data to 79. The enrollment process was assisted by a trained interviewer. Detailed recruitment strategies for NEST have been described in a previous study [23]. English speaking pregnant mothers delivered between July 2005 and November 2006 at Duke University Hospital, Durham, NC.

\section{Data collection}

The questionnaire included maternal socio-demographic data, such as age, marital status, race and education, as well as multiple lifestyle or health characteristics (for example, smoking, chronic diseases). One of the main items of the survey included a detailed list of questions about the mother's and the father's height, highest and lowest weight ever, current and usual weight. Medical records were used to verify medical conditions, and to abstract gestation time, birth weight and the newborn's gender. Body mass index was calculated from the data obtained from height and the mother's weight before pregnancy or the father's current weight. Obesity was defined as BMI $\geq 30 \mathrm{~kg} / \mathrm{m}^{2}$.

\section{Specimen collection}

At delivery, umbilical cord blood samples were collected in a vacuum blood collection tube coated with $\mathrm{K}_{3}$ EDTA. The tubes were centrifuged to isolate the buffy coat; this leukocyte-containing buffy coat was then stored at 
$-80^{\circ} \mathrm{C}$. Genomic DNA was extracted using Gentra Puregene Reagents (Qiagen, Valencia, CA, USA).

\section{DNA methylation analysis}

IGF2 and H19 DMRs were analyzed by pyrosequencing. The IGF2 DMR includes three CpG dinucleotides upstream of IGF2 exon 3 (chr 11p15.5; CpG site 1: 2,169,518; CpG site 2: 2,169,515; and CpG site 3: 2,169,499; NCBI Human Genome Build 37/hg19). This DMR has been previously evaluated by Cui et al. [6] and Heijmans et al. [8]. The region studied for the H19 DMR encompasses four dinucleotides located upstream of the H19 gene (chr 11p15.5; CpG site 1: 2,024,261, CpG site 2: 2,024,259, CpG site 3: 2,024,257, and CpG site 4: 2,024,254; NCBI Human Genome Build 37/hg19), which is the first of six known sequence motifs that bind the CTCF zinc finger protein [24,25]. The structural characteristics of these IGF2 and H19 loci and the genomic coordinates of the assays have been presented elsewhere [7,26]. Genomic DNA (800 ng) was treated with sodium bisulfite [27], the IGF2/H19 regions were amplified by PCR, and pyrosequencing was performed in duplicate using a Pyromark Q96 MD pyrosequencing instrument (Qiagen). Control assays were also run to validate our methylation results. Detailed methodology, including assay conditions and validation studies, have been described previously [25].

\section{Statistical methods}

Variables were defined as follows: race (African American, Caucasian, and other), age (as a continuous variable), marital status (living with partner versus single), at least a college graduate (yes or no), pre-pregnancy maternal and paternal obesity $\left(<30\right.$ versus $\left.\geq 30 \mathrm{~kg} / \mathrm{m}^{2}\right)$, smoking status (never/quit smoking when pregnant/continued smoking during pregnancy), birth weight of the baby $(<2.5 \mathrm{~kg} /$ between $2.5 \mathrm{~kg}$ and $3.5 \mathrm{~kg} / \geq 3.5 \mathrm{~kg})$, gestation time $(<37$ weeks versus $\geq 37$ weeks), and gender of the baby (male or female) (Table 1). Chi Square tests were used to compare obesity of the mother and the father within different subgroups of pregnant women. If numbers were small $(<5)$ the Fisher exact test was used. We also compared the characteristics for missing and non-missing experimental methylation results and for missing and non-missing BMI of the father. Methylation levels were distributed normally in the groups studied (verified by using the KolmogorovSmirnov test). Student's $t$-tests were computed to test for significant differences in the methylation means. We assessed the effect of maternal and paternal obesity (BMI $\geq 30 \mathrm{~kg} / \mathrm{m}^{2}$ ) on the methylation levels of the IGF2 and H19 DMRs, which were analyzed separately by individual CpG site, as well as by the mean of the CpGs. To account for batch effects from the laboratory tests on different days or different plates we calculated the least square means (or
Table 1 Parental and newborn characteristics

\begin{tabular}{|c|c|c|c|}
\hline \multicolumn{2}{|c|}{ NEST - Newborn Epigenetics Study Cohort 2005 to 2006} & \multirow{2}{*}{$\begin{array}{c}n \\
59\end{array}$} & \multirow{2}{*}{$\frac{\%}{67.8}$} \\
\hline BMI mother: & BMI $<30$ (not obese) & & \\
\hline & $\mathrm{BMI} \geq 30$ (obese) & 28 & 32.2 \\
\hline \multirow[t]{2}{*}{ BMI father: } & BMI <30 (not obese) & 63 & 79.7 \\
\hline & $\mathrm{BMI} \geq 30$ (obese) & 16 & 20.3 \\
\hline \multirow[t]{2}{*}{ Marital status: } & Living with partner & 72 & 74.2 \\
\hline & Single & 25 & 25.8 \\
\hline \multirow[t]{2}{*}{ Education: } & Low (no college degree) & 57 & 58.8 \\
\hline & High (at least college degree) & 40 & 41.2 \\
\hline \multirow[t]{3}{*}{ Race: } & African American & 38 & 38.8 \\
\hline & Caucasian & 56 & 57.1 \\
\hline & Other or not specified & 4 & 4.08 \\
\hline \multirow[t]{2}{*}{ Maternal age: } & $<30$ years & 56 & 57.1 \\
\hline & $\geq 30$ years & 42 & 42.9 \\
\hline \multirow[t]{3}{*}{ Smoking: } & Mother never smoked & 45 & 48.4 \\
\hline & Quit smoking when pregnant & 26 & 27.9 \\
\hline & Smoked during pregnancy & 22 & 23.7 \\
\hline \multirow[t]{2}{*}{ Gestation time: } & Preterm ( $<37$ weeks) & 10 & 10.3 \\
\hline & Normal ( $\geq 37$ weeks) & 87 & 86.7 \\
\hline \multirow[t]{2}{*}{ Birth weight: } & $<2.5 \mathrm{~kg}$ & 16 & 16.5 \\
\hline & $\geq 2.5 \mathrm{~kg}$ & 81 & 83.5 \\
\hline \multirow[t]{2}{*}{ Baby gender: } & Male & 48 & 49.5 \\
\hline & Female & 49 & 50.5 \\
\hline
\end{tabular}

This sub-cohort includes all NEST families from whom babies were born at Duke University Hospital between July 2005 and November 2006. Characteristics of mothers, fathers and newborns are shown for the 98 participants when data were not missing.

estimated marginal means) of each CpG site. We evaluated potential confounding for paternal and maternal obesity by means of multivariable regression analyses. We used multiple regression analyses, separately for each exposure and characteristics from Table 1. These multiple regression analyses were used to predict DNA methylation at each DMR site. To further explore whether the association between obesity and DNA methylation varied by race, we repeated these analyses in African Americans and Caucasians. Graphs representing the associations between BMI and methylation outcomes were based on the predicted outcomes of the methylation means, after adjusting for maternal age, smoking status, birth weight and gender. Observations included in the regression analyses were determined by the availability of laboratory measures for the IGF2 and H19 DMRs. All statistical analyses were conducted in SAS v9.2 (SAS Institute Inc., Cary, NC, USA), and GraphPad Prism 5 was used to obtain Figures 1 and 2 (GraphPad Software Inc., La Jolla, CA, USA).

\section{Ethics}

The experimental research in this report was performed with the approval of the Duke University Institutional Review Board, reference number: Pro00014548; this means that the research carried out is in compliance with 


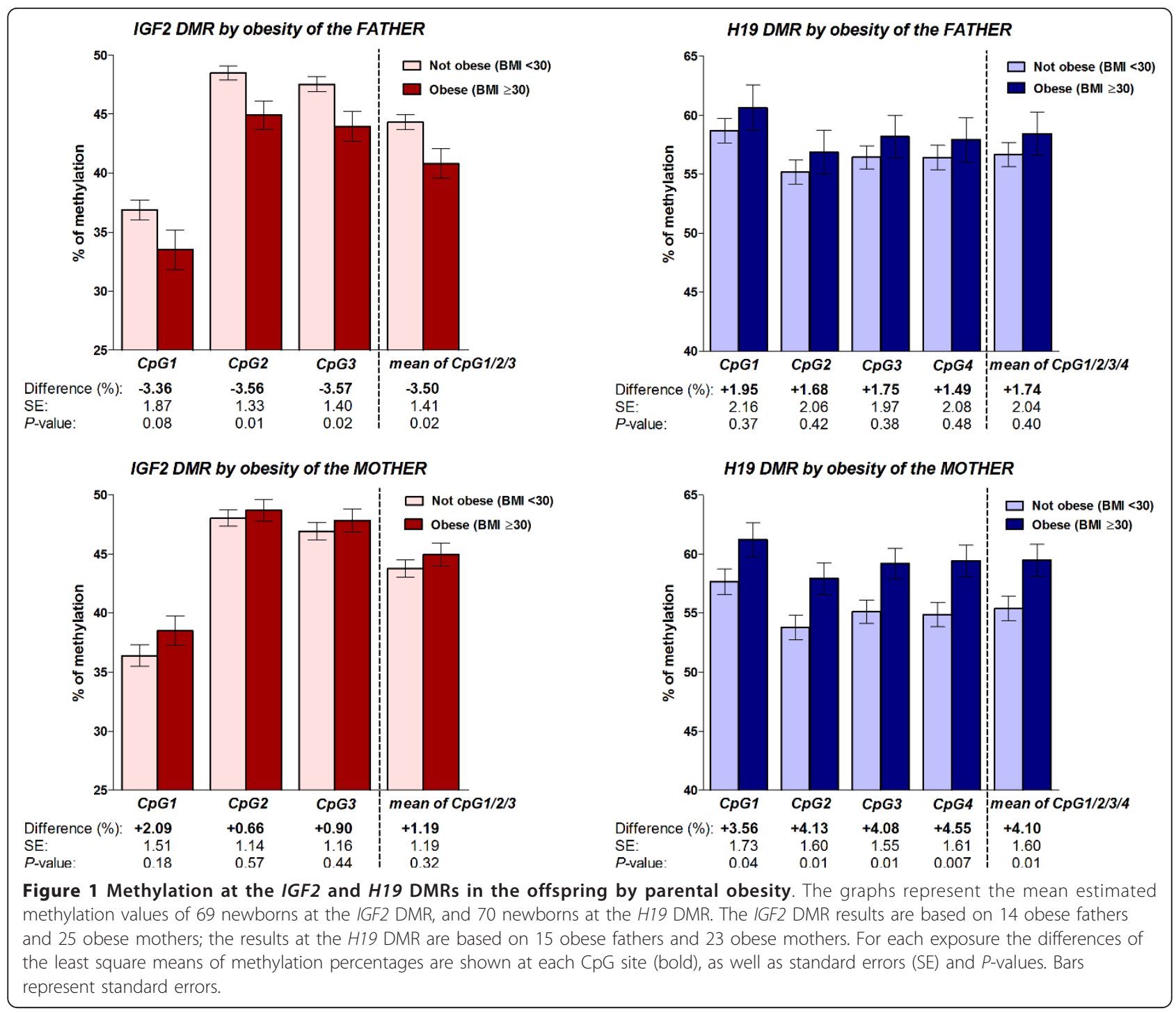

the Helsinki Declaration. All participants signed the informed consent for the use of their medical record data, questionnaire data, their newborn's birth parameters and umbilical cord blood.

\section{Results}

\section{Characteristics of study participants}

The distributions of socio-demographic data are shown in Table 1. Seventy-four percent of the mothers were living with a partner, and nearly $26 \%$ were single. The majority of the study population were Caucasians (57.1\%) or African Americans (38.8\%), and the other races/ethnicities included Asians, Native Americans or non-specified race $(4.1 \%)$. A quarter of the mothers were between 25 and 29 years old (26.5\%). Nearly 59\% never obtained a college degree, and were categorized as "low education". Approximately $24 \%$ of the mothers reported smoking during pregnancy, and 32\% were obese before pregnancy. About $80 \%$ of the mothers provided data about the father's characteristics: $20 \%$ are obese. Approximately $16 \%$ of the newborns had a low birth weight, $10 \%$ was born preterm and gender was equally distributed. None of the included characteristics were associated with paternal obesity, with the exception of obesity in the mother $(P=0.01)$. For instance, potential associations between birth weight and parental obesity were excluded by Chi Square tests or Fisher exact tests; $P$-values were 0.7 for paternal and maternal obesity. Maternal obesity was positively associated with single motherhood $(P=$ 0.002), being African American $(P<0.001)$ and being a non-smoker $(P=0.06)$. Given that $19 \%$ of our subjects did not report detailed information about the fathers, we compared all measured characteristics and methylation end-results in newborns with missing anthropometric 

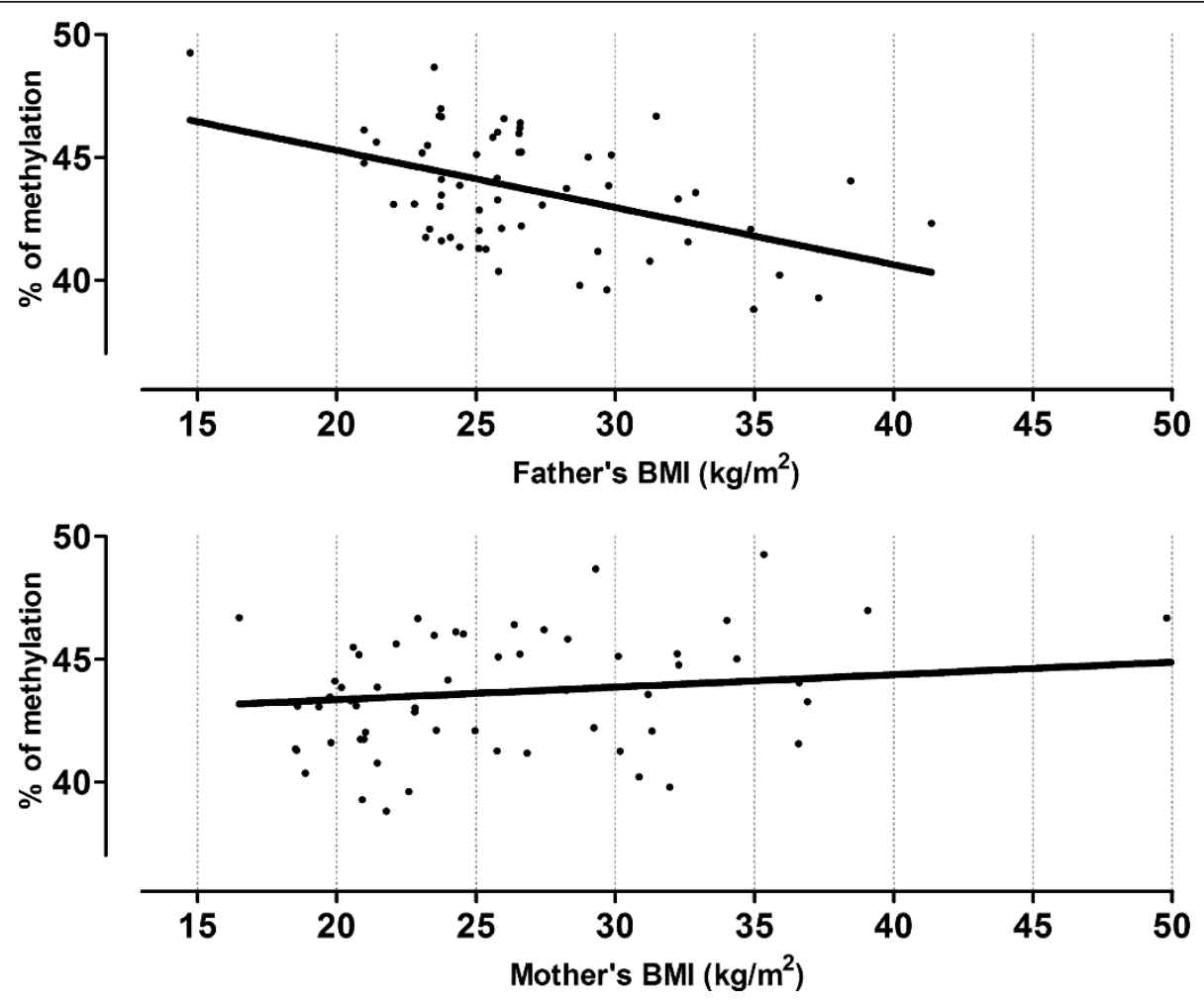

Figure 2 Offspring's mean methylation \% at the IGF2 DMR by BMI of the parents. The predicted methylation means at IGF2 DMR are plotted by BMI of the father (upper graph), and BMI of the mother (lower graph); adjusted for maternal age, smoking status, BMI of the other parent, the newborn's birth weight and gender.

data about fathers versus those without missing data. The majority of the missing paternal data (78\%) were from African American mothers with lower education. Single mothers were less likely to answer questions about the father's weight and height (32.0\%), compared with married mothers or those living with a partner (13.9\%), $P=$ 0.07. Other characteristics and the methylation outcomes did not differ significantly between both groups.

\section{Associations between paternal or maternal obesity and methylation profiles at the IGF2 and H19 DMRs in the offspring}

Using DNA extracted from umbilical cord blood leukocytes we determined the levels of methylation at CpG sites within the IGF2 and H19 DMRs in newborns. From the 79 samples in our cohort, we obtained experimental data for 67 participants at the IGF2 DMR and for 70 participants at the H19 DMR. We further refer to these participants as our analytical cohort. No significant differences in characteristics were found when comparing the missing with the non-missing experimental methylation results for both DMRs. We analyzed methylation outcomes by the characteristics described in Table 1 and with the exception of obesity (BMI $\geq 30 \mathrm{~kg} / \mathrm{m}^{2}$ ), none showed significant associations with methylation percentages at the IGF2 or H19
DMRs in our cohort. Figure 1 shows the estimated means of methylation percentages by obesity of the parents. Differences in methylation percentages among offspring from obese fathers compared to non-obese fathers at CpG1, CpG2, and CpG3 of the IGF2 DMR were: $-3.36 \%$ $(P=0.08),-3.56 \%(P=0.01)$ and $-3.57 \%(P=0.02)$, respectively; the mean methylation difference was: $-3.50 \%(P=$ 0.02 ) (Figure 1). Methylation in offspring among obese fathers was significantly lower than when compared to fathers who were not obese. We observed no differences in methylation percentages at the IGF2 DMR between offspring of obese and non-obese mothers. Differences in methylation percentages at CpG1, CpG2 and CpG3 were $+2.09 \%(P=0.18),+0.66 \%(P=0.57)$ and $+0.90 \%(P=$ $0.44)$, respectively; the mean methylation difference was $+1.19 \%(P=0.32)$ (Figure 1).

At the H19 DMR we found no methylation differences in the offspring of obese compared to non-obese fathers. Differences in methylation percentages at CpG1, CpG2, CpG3 and CpG4 of the H19 DMR were $+1.95 \%(P=$ $0.37),+1.68 \%(P=0.42),+1.75 \%(P=0.38)$ and $+1.49 \%$ $(P=0.48)$, respectively; the mean methylation difference was $+1.74 \%(P=0.40)$ (Figure 1$)$. However, the methylation percentages at the CpG sites of the H19 DMR among offspring from obese mothers were significantly 
higher at CpG1, CpG2, CpG3 and CpG4 compared to offspring from non-obese mothers. The differences were $+3.56 \%(P=0.04),+4.13 \%(P=0.01),+4.08 \%(P=0.01)$ and $+4.55 \%(P=0.007)$, respectively; the mean methylation difference was $+4.10 \%(P=0.01)$ (Figure 1$)$. In brief, without adjusting for potential confounders we detected significantly lower methylation at the IGF2 DMR associated with paternal obesity, and significantly higher methylation at the H19 DMR when the mother was obese.

To assess the independent effects of preconceptional maternal or paternal obesity on DNA methylation at the IGF2 and H19 DMRs in newborns, we used multiple regression models, controlling for potential confounders, including maternal age, maternal smoking status, gender of the baby, birth weight and experimental batch effects. The results of the regression analyses, found in Table 2, show that paternal obesity is inversely associated with DNA methylation levels at the IGF2 DMR in newborns. Controlling for potential confounding did not change this relationship. After adjusting for several characteristics, we observed a significant decrease in methylation when fathers were obese, $\beta$-coefficient was -5.28 ( $P=$ 0.003) (Table 2, Model 3). Little difference for this association was observed when comparing the individual CpG sites. The $\beta$-coefficients for CpG1, CpG2 and CpG3 were $-5.78(P=0.01),-5.18(P=0.002)$ and -4.76 $(P=0.007)$, respectively. In contrast, results for maternal obesity indicated an increase in methylation at the IGF2 DMR, the $\beta$-coefficient was $+3.08(P=0.05)$ when controlling for the same characteristics (Table 2, Model 3). The $\beta$-coefficients at the individual $\mathrm{CpG}$-sites were $+4.23(P=0.04),+2.44(P=0.08),+2.42(P=0.12)$, for CpG1, CpG2 and CpG3; with only CpG1 reaching statistical significance.

In our separate regression models by maternal and paternal obesity, we observed a positive trend in DNA methylation at the H19 DMR when adjusting for maternal and newborn characteristics (Table 2, Models 1 and 2 , respectively). The $\beta$-coefficient was +3.09 when the fathers were obese, but this result was not significant

Table 2 Linear Regression Models: methylation at the IGF2 and H19 DMRs in relation to parental obesity

\begin{tabular}{lcccccccc}
\hline Linear regression models & \multicolumn{3}{c}{ IGF2 DMR } & \multicolumn{3}{c}{ H19 DMR } \\
\hline & Obesity of: & $\boldsymbol{\beta}$ & SE & $\boldsymbol{P}$ & $\boldsymbol{\beta}$ & SE & $\boldsymbol{P}$ \\
Model 1 & Father & $\mathbf{- 3 . 8 3}$ & 1.48 & 0.01 & $\mathbf{+ 3 . 0 9}$ & 1.64 & 0.07 \\
Model 2 & Mother & $\mathbf{+ 2 . 3 8}$ & 1.30 & 0.08 & $\mathbf{+ 2 . 8 0}$ & 1.38 & 0.05 \\
Model 3 & Father & $\mathbf{- 5 . 2 8}$ & 1.62 & 0.003 & $\mathbf{+ 2 . 5 5}$ & 1.82 & 0.17 \\
& Mother & $\mathbf{+ 3 . 0 8}$ & 1.48 & 0.05 & $\mathbf{+ 1 . 0 5}$ & 1.70 & 0.54 \\
\hline
\end{tabular}

Obesity was defined as BMI $\geq 30 \mathrm{~kg} / \mathrm{m}^{2}$. All models were adjusted for maternal age and smoking status, as well as by the newborn's birth weight and gender. Models 1 and 2 include either maternal or paternal obesity. Model 3 includes both maternal and paternal obesity.
$(P=0.07)$, and +2.80 when the mothers were obese $(P=0.05)$. The multivariate model with the mean methylation percent as a dependent variable and several measured maternal, paternal and newborn characteristics as independent variables (Model 3 ) showed a $\beta$ coefficient for offspring from obese fathers of $+2.55(P=$ 0.17 ), and a $\beta$-coefficient for offspring from obese mothers of $+1.05(P=0.54)$. Similar results were seen when we evaluated the individual CpG sites (data not shown).

We further stratified our analytical cohort by race (Caucasian and African American) and repeated all regression analyses described above. At the IGF2 DMR, the outcome was similar as in our complete analytical cohort. We calculated a $\beta$-coefficient of -5.16 in offspring from obese fathers, among Caucasians $(P=0.01)$ (as in Model 3); and a $\beta$-coefficient of -3.65 in offspring from obese fathers, among African Americans. Although the estimates suggest an inverse relationship in Caucasian subjects, this effect was not significant among African Americans $(P=0.34)$. These observations were based on only 10 obese fathers in Caucasians, and only 4 obese fathers in African Americans. At the H19 DMR, results were also similar and in the same direction as those in our complete analytical cohort. However, we found one higher estimate in Model 1 for children from African American obese fathers: $\beta$-coefficient was $+8.14(P=$ 0.007). But, this result was based on a small sample size with only 6 obese African American fathers. Together with our statistical power calculations we conclude that the stratified analyses represent unstable estimates.

We further extended the regression analyses in our analytical cohort by replacing the dichotomous obesity variable by the continuous BMI variable. At the H19 DMR, we found a $\beta$-coefficient of $+0.21(P=0.12)$ for paternal BMI and $+0.16(P=0.09)$ for maternal BMI, controlling for the same variables as before; but these results were still not significant. Whereas at the IGF2 DMR, our data reached a significant positive association when both paternal and maternal BMI were used as independent variables in the multivariate model; $\beta$-coefficients were $-0.38(P=0.009)$, and $+0.22(P=0.02)$, respectively. These correlations between parental BMI and the predicted mean methylation levels at the IGF2 DMR in the offspring are depicted in Figure 2.

\section{Discussion}

We explored the potential effect of parental obesity on IGF2/H19 DMR methylation in newborns. We found a significant decrease in methylation among newborns of obese fathers at the IGF2 DMR in DNA extracted from cord blood leucocytes. This finding remained significant after controlling for potential confounders $(\beta$-coefficient $=$ -5.28, $P=0.003)$. Hypomethylation at the IGF2 DMR has 
been associated with an increased risk of developing cancers, such as Wilms' tumor [28], colorectal cancer [6] and ovarian cancer [7]. We found no significant changes in methylation levels associated with paternal obesity at the H19 DMR region.

Obesity is a metabolic condition that has paradoxically been associated with poverty, low quality of life, malnutrition and an imbalanced intake of nutrients [21,22]. Few epidemiological studies indicate potential correlations between obesity or food supplies in the paternal line and offspring's birth weight [29], body-fat in prepubertal girls [30], or mortality from chronic diseases [31-33]. Epidemiological data regarding associations between maternal obesity and the offspring's birth weight vary by study (reviewed by McDonald et al. [34]). We examined possible associations between paternal or maternal obesity and birth weight but detected no associations. Epidemiological studies on maternal obesityrelated exposures generally show a positive association between abnormally high BMI and congenital anomalies (reviewed by Stothard et al. [35]). These harmful effects are mostly attributed to in utero exposures to malnutrition or overnutrition; while very often, data indicate the importance of exposures at the very early stages of development, even before conception. Research in animal models suggests that potential diet-dependent transgenerational effects may be explained by changes in the establishment of epigenetic gene regulatory marks [12,36-38]. Analyses of adults born to mothers exposed to poor nutrition during the Dutch famine indicated a $5 \%$ decrease in methylation at the IGF2 DMR compared to the same sex non-exposed siblings. Interestingly, the magnitude of this effect is similar to the effect we observe in offspring from obese fathers at the same locus. In the Dutch famine cohort, exposure during the periconceptional period was more important than during the second or the third trimester of gestation [8]. Furthermore, not only do exposures during early gestation cause harmful health outcomes, but famine prior to conception has also been associated with poor health [39]. Similar results were found among Gambian children; they exhibit altered DNA methylation at several metastable epialleles according to the seasonal nutritional circumstances in which they were conceived [40]. Both cohorts described above did not directly examine the relationship with the fathers' dietary patterns; although they were most likely exposed to the same famine or nutritional circumstances as the mothers. Analysis of the Framingham Heart Study indicates that early-onset paternal obesity, and not maternal obesity, increases the odds of aberrant serum levels of the metabolic biomarker ALT (alanine transaminase) in the offspring [41]. Studies on animal models have shown similar associations. In rats, a high fat paternal diet results in offspring with early onset of impaired insulin secretion, altered expression of multiple genes related to normal pancreatic beta-cell function, and altered methylation at a putative regulatory region of the Interleukin 13 receptor, alpha 2 gene [42]. Male mice whose mothers were exposed to a high-fat diet were not only obese, insulin insensitive and diabetic, they were also capable of passing part of this phenotype to the next generation, suggesting an underlying epigenetic mechanism transmitted through germ cells [43]. To our knowledge, our study is the first epidemiological study that suggests a similar underlying epigenetic mechanism conferred by harmful paternal dietary patterns or obesity.

Obesity is associated with elevated IGF2 circulating levels [44] and increased estrogen levels [45]. Although we did not include IGF2 protein levels in parents or offspring in our current analyses, we earlier showed that hypomethylation at the IGF2 DMR is associated with higher circulating IGF2 levels in the offspring $[25,46]$. This association was strongest in offspring from obese mothers, independent of race. In brief, a decrease of $5 \%$ at the $I G F 2$ DMR methylation corresponded to an increase of at least $10 \%$ in serum concentration of IGF2 [46]. In addition, other studies have shown that small aberrant methylation changes at the IGF2 or H19 DMR is linked to increased expression of IGF2 $[6,7,24,25,47]$, as well as an increased susceptibility to chronic diseases [48-51]. Similar small effects on DNA methylation have also been associated with the use of assisted reproductive technologies [52], the use of psychotropic drugs during pregnancy [53] and smoking [25]. These subtle epigenetic changes have been described as adaptive responses to the environment, while major epigenetic shifts during development would cause more detrimental consequences [25]. Furthermore, exploring the CpG sites at the IGF2 and H19 DMRs may represent only a fraction of changes occurring elsewhere in the genome. Environmental factors, among which is diet, have been associated with changes in DNA methylation and may have profound effects on genomic imprinting; accumulation of these effects may result in disturbed metabolic homeostasis [54]. Follow-up studies on the anthropometric and other developmental factors of the NEST children are underway to further examine the influence of small changes in DNA methylations at several DMRs on childhood obesity or other adverse consequences. Evidence in animal studies indicates that DNA methylation at the IGF2/H19 locus in sperm might be under tight control of estrogen [55], produced by adipocytes. This suggests a mechanism by which increased exposure to estrogen could lead to inadequate establishment of methylation at the IGF2 DMR in sperm. Alternatively, obesity-related factors may also disrupt functioning of other components of the epigenetic machinery leading to an inability to appropriately establish imprint marks during spermatogenesis, which is ongoing through adult male life [5]. 
Offspring of obese fathers may, therefore, demonstrate incomplete methylation. In order to further explore these hypotheses, more research on the epigenetic effects of obesity on human germ cells is needed.

Although our bivariate analysis did not indicate an association between maternal obesity and DNA methylation at the IGF2 DMR in newborns, the regression analysis showed that controlling for paternal obesity resulted in an opposite effect, meaning that while paternal obesity was associated with a decrease in methylation, maternal obesity tended to be associated with an increase in methylation, but this was only significant at one CpG site. Using pre-pregnancy BMI instead of obesity in our multivariate analysis strengthened this association. At the $H 19$ DMR, the bivariate analysis showed an increase of $4.1 \%$ when mothers were obese $(P=0.01)$. An increase in DNA methylation by maternal pre-gestational BMI has also been reported earlier in cord blood samples, more particularly at the PPARG promoter [56]. Hypermethylation at the IGF2 or H19 DMR has been associated with loss of imprinting of $I G F 2$, and several disorders. For instance, IGF2 imprinting defects have been implicated in Silver-Russell syndrome [57], Wilms' tumor [24,58], hepatoblastoma [59] and ovarian cancer [7]. Our regression analyses at the H19 DMR showed that adjusting for potential confounders, including paternal obesity or BMI, diminished this positive association (Table 2, Model 3). We attribute this to the fact that maternal and paternal obesity are closely related and the fact that methylation outcomes for both parental exposures are in the same direction. A larger study is necessary to further explore the potential impact of parental obesity on DNA methylation at the H19 DMR. Furthermore, the exposure from oocyte stage till birth to maternal obesity or related lifestyles is complex. Hormonal factors that may influence DNA methylation cannot be ruled out. It has been shown that the rat H19 DMR has an estrogen responsive element, suggesting that estrogen can form a complex with Dnmt1, a DNA methyl transferase, leading to DNA methylation at a normally unmethylated maternal allele [55].

A potential limitation of our study includes the use of cord blood as a marker for the newborn's epigenetic status. However, we used isolated leucocytes and IGF2 is a well studied imprinted gene whose germline DMRs should be similarly methylated across all cell types, given the establishment of the epigenetic profile prior to conception. IGF2 and H19 DMR methylation profiles were verified in DNA from different cell fractions from umbilical cord blood and we found no differences across the cell types [26]. Another possible limitation is proof of paternity, and the reliability of the paternal anthropometric data, which were reported by pregnant mothers. However, the questions regarding the anthropometric data were detailed and verified for consistency. We do not expect that methylation outcomes are differential with respect to the potential misclassification of exposures. Nineteen percent of our population had missing data about the father. The methylation outcomes of these newborns were not included in our final analytical study group. However, we compared methylation outcomes by missing and non-missing characteristics of the fathers and found no differences. We also compared all measured maternal and newborn characteristics in both groups and most characteristics were similarly distributed. We found no significant differences when mothers were single or not, but found significant differences by education and race. Most missing data were among the lower educated and African American mothers. As far as we could test, education was not associated with obesity in either of the parents, and race was only associated with obesity of the mother. It is very likely we missed a number of obese fathers from African Americans. We cannot verify if paternal obesity is equally distributed in all subgroups of missing and non-missing paternal data, and, therefore, selection bias cannot be excluded. However, when reanalyzing our regression models with race included as an independent variable, our results remained the same; only the effect of maternal obesity attenuated in Model 2 (Table 2) at H19 DMR, the $\beta$-coefficient for maternal obesity became $+2.18(\mathrm{SE}=1.65, P=$ 0.19) (data not shown). Possibly, race is on the same causal pathway as obesity, regarding the effect on methylation outcomes. As mentioned in the results section, race by itself was not associated with methylation outcomes at neither of the two DMRs studied in this cohort. Nevertheless, given our earlier analyses on maternal exposures showed a race dependent effect at the same imprinted loci [53], we further stratified our data by race. The outcomes were in the same direction as the complete analytical cohort, but given the smaller numbers, the results represented unstable estimates. In general, the small sample size remains a limitation in our study which may partly explain why our results at the H19 DMR do not reach statistical significance. Nevertheless, the data concerning associations between IGF2 DMR methylation and parental BMI or obesity reached sufficient power, especially when studying offspring from fathers with high BMI.

\section{Conclusions}

Our early findings suggest that lifestyle factors of parents may be indirectly transmitted to the next generation via epigenetic mechanisms. We show that one of the measurable lifestyle parameters, obesity of the father, is associated with hypomethylation at the IGF2 DMR in the offspring. Aberrant low methylation at this DMR has been associated with adverse health outcomes. As a 
consequence, our preliminary result may be important for public health, especially in regard to the global burden of obesity. We hypothesize that the molecular mechanism behind our observation might be a hormonal difference between obese and non-obese parents, inducing an incomplete or unstable establishment of methylation at the IGF2 DMR during gametogenesis. As a result, exposures to adverse lifestyle factors or poor/ over-nutrition during spermatogenesis may affect the reprogramming of methylation profiles at imprinted genes. Further research is necessary to confirm this hypothesis. Although our study population is small, this is the first report in humans suggesting that paternal obesity may disrupt the normal establishment of genomic imprinting in germ cells.

\section{Abbreviations}

BMI: body mass index; DMRs: differentially methylated regions; ICR: imprinting control region; IGF2: Insulin-like Growth Factor 2; NEST: Newborn Epigenetics Study

\section{Authors' contributions}

AS developed the hypothesis of this study, designed the analytical strategy, analyzed the data and wrote the manuscript. JS contributed to the analysis and interpretation of the data, and helped to draft the manuscript. AM oversaw participant recruitment in the clinic and contributed to editing the manuscript. FW implemented the statistical analysis. ZH performed the assays. $A B$ contributed to the research discussions and the editing of the manuscript. JK contributed to the logistics of data collection. RJ contributed to the inception of the original NEST research hypothesis. SM is co-principal investigator and oversaw laboratory analysis and processing of the specimens and helped to draft the manuscript. $\mathrm{CH}$ is the principle investigator who oversaw the design and conduct of NEST. All authors have read the manuscript and given their final approval of submission for publication.

\section{Competing interests}

The authors declare that they have no competing interests.

\section{Acknowledgements}

We thank the participants of the Newborn Epigenetics Study. We also thank the NEST project coordinator Stacy Murray, the data manager Francine Overcash, the research nurse Tammy Bishop, and the laboratory technicians Carole Grenier, Darby Kroyer, Erin Erginer, Cara Davis and Allison Barratt. This work was supported by National Institutes of Health (R21ES014947, R01ES016772, R01DK085173, R25CA126938-01A2), the American Cancer Society (ACS-IRG 83-006), Fulbright, and the Fred and Alice Stanback Foundation.

\section{Author details}

'Duke Cancer Institute, Duke University Medical Center 2715, Durham, NC 27710, USA. ${ }^{2}$ Department of Community and Family Medicine, Duke University Medical Center 104006, Durham, NC 27710, USA. ${ }^{3}$ Division of Maternal and Fetal Medicine, Department of Obstetrics and Gynecology, Duke University Medical Center, 4022 Hospital South, Durham, NC 27705, USA. ${ }^{4}$ Department of Obstetrics and Gynecology, Division of Gynecologic Oncology, Duke University Medical Center 91012, Durham, NC 27708, USA. ${ }^{5}$ Department of Radiation Oncology, Duke University Medical Center 3433, Durham, NC 27710, USA. 'Department of Pediatrics, Duke University Medical Center 3350, Durham, NC 27710, USA. 7 Department of Obstetrics and Gynecology, Division of Clinical Epidemiology, Duke University Medical Center 2914, Durham, NC 27710, USA.

Received: 19 April 2012 Accepted: 6 February 2013 Published: 6 February 2013
References

1. McKay JA, Mathers JC: Diet induced epigenetic changes and their implications for health. Acta Physiol (Oxf) 2011, 202:103-118.

2. Baker J, Liu JP, Robertson EJ, Efstratiadis A: Role of insulin-like growth factors in embryonic and postnatal growth. Cell 1993, 75:73-82.

3. Chao W, D'Amore PA: IGF2: epigenetic regulation and role in development and disease. Cytokine Growth Factor Rev 2008, 19:111-120.

4. Boissonnas CC, Abdalaoui HE, Haelewyn V, Fauque P, Dupont JM, Gut I, Vaiman $D$, Jouannet $P$, Tost J, Jammes $H$ : Specific epigenetic alterations of IGF2-H19 locus in spermatozoa from infertile men. Eur J Hum Genet 2010, 18:73-80.

5. Kerjean A, Dupont JM, Vasseur C, Le Tessier D, Cuisset L, Paldi A, Jouannet $P$, Jeanpierre $M$ : Establishment of the paternal methylation imprint of the human $\mathrm{H} 19$ and MEST/PEG1 genes during spermatogenesis. Hum Mol Genet 2000, 9:2183-2187.

6. Cui $\mathrm{H}$, Onyango $\mathrm{P}$, Brandenburg $\mathrm{S}, \mathrm{Wu}$ Y, Hsieh $\mathrm{CL}$, Feinberg AP: Loss of imprinting in colorectal cancer linked to hypomethylation of $\mathrm{H} 19$ and IGF2. Cancer Res 2002, 62:6442-6446.

7. Murphy SK, Huang Z, Wen Y, Spillman MA, Whitaker RS, Simel LR, Nichols TD, Marks JR, Berchuck A: Frequent IGF2/H19 domain epigenetic alterations and elevated IGF2 expression in epithelial ovarian cancer. Mol Cancer Res 2006, 4:283-292.

8. Heijmans BT, Tobi EW, Stein AD, Putter H, Blauw GJ, Susser ES, Slagboom PE, Lumey LH: Persistent epigenetic differences associated with prenatal exposure to famine in humans. Proc Natl Acad Sci USA 2008, 105:17046-17049

9. Steegers-Theunissen RP, Obermann-Borst SA, Kremer D, Lindemans J, Siebel C, Steegers EA, Slagboom PE, Heijmans BT: Periconceptional maternal folic acid use of 400 microg per day is related to increased methylation of the IGF2 gene in the very young child. PLoS One 2009, 4 e7845.

10. Ollikainen M, Smith KR, Joo EJ, Ng HK, Andronikos R, Novakovic B, Abdul Aziz NK, Carlin JB, Morley R, Saffery R, Craig JM: DNA methylation analysis of multiple tissues from newborn twins reveals both genetic and intrauterine components to variation in the human neonatal epigenome. Hum Mol Genet 2010, 19:4176-4188.

11. Ravelli GP, Stein ZA, Susser MW: Obesity in young men after famine exposure in utero and early infancy. N Engl J Med 1976, 295:349-353.

12. Waterland RA, Jirtle RL: Transposable elements: targets for early nutritional effects on epigenetic gene regulation. Mol Cell Biol 2003, 23:5293-5300.

13. Barker DJ: Intrauterine programming of coronary heart disease and stroke. Acta Paediatr Supp/ 1997, 423:178-182, discussion 183.

14. Dolinoy DC, Jirtle RL: Environmental epigenomics in human health and disease. Environ Mol Mutagen 2008, 49:4-8.

15. Painter RC, de Rooij SR, Bossuyt PM, Simmers TA, Osmond C, Barker DJ, Bleker OP, Roseboom TJ: Early onset of coronary artery disease after prenatal exposure to the Dutch famine. Am J Clin Nutr 2006, 84:322-327, quiz 466-327.

16. Painter RC, De Rooij SR, Bossuyt PM, Osmond C, Barker DJ, Bleker OP, Roseboom TJ: A possible link between prenatal exposure to famine and breast cancer: a preliminary study. Am J Hum Biol 2006, 18:853-856.

17. Dolinoy DC, Weidman JR, Jirtle RL: Epigenetic gene regulation: linking early developmental environment to adult disease. Reprod Toxicol 2007, 23:297-307.

18. Waterland RA, Jirtle RL: Early nutrition, epigenetic changes at transposons and imprinted genes, and enhanced susceptibility to adult chronic diseases. Nutrition 2004, 20:63-68.

19. Levin BE: Metabolic imprinting: critical impact of the perinatal environment on the regulation of energy homeostasis. Philos Trans $R$ SoC Lond B Biol Sci 2006, 361:1107-1121.

20. Boerschmann H, Pfluger M, Henneberger L, Ziegler AG, Hummel S: Prevalence and predictors of overweight and insulin resistance in offspring of mothers with gestational diabetes mellitus. Diabetes Care 2010, 33:1845-1849.

21. Dyer JS, Rosenfeld CR: Metabolic imprinting by prenatal, perinatal, and postnatal overnutrition: a review. Semin Reprod Med 2011, 29:266-276.

22. Tanumihardjo SA, Anderson C, Kaufer-Horwitz M, Bode L, Emenaker NJ, Haqq AM, Satia JA, Silver HJ, Stadler DD: Poverty, obesity, and malnutrition: an international perspective recognizing the paradox. J Am Diet Assoc 2007, 107:1966-1972. 
23. Hoyo C, Murtha AP, Schildkraut JM, Forman MR, Calingaert B, DemarkWahnefried W, Kurtzberg J, Jirtle RL, Murphy SK: Folic acid supplementation before and during pregnancy in the Newborn Epigenetics STudy (NEST). BMC Public Health 2011, 11:46.

24. Cui H, Niemitz EL, Ravenel JD, Onyango P, Brandenburg SA, Lobanenkov W, Feinberg AP: Loss of imprinting of insulin-like growth factor-II in Wilms' tumor commonly involves altered methylation but not mutations of CTCF or its binding site. Cancer Res 2001, 61:4947-4950.

25. Murphy SK, Adigun A, Huang Z, Overcash F, Wang F, Jirtle RL, Schildkraut JM, Murtha AP, Iversen ES, Hoyo C: Gender-specific methylation differences in relation to prenatal exposure to cigarette smoke. Gene 2012, 494:36-43

26. Murphy SK, Huang Z, Hoyo C: Differentially methylated regions of imprinted genes in prenatal, perinatal and postnatal human tissues. PLoS One 2012, 7:e40924.

27. Huang Z, Wen Y, Shandilya R, Marks JR, Berchuck A, Murphy SK: High throughput detection of M6P/IGF2R intronic hypermethylation and LOH in ovarian cancer. Nucleic Acids Res 2006, 34:555-563.

28. Sullivan MJ, Taniguchi T, Jhee A, Kerr N, Reeve AE: Relaxation of IGF2 imprinting in Wilms tumours associated with specific changes in IGF2 methylation. Oncogene 1999, 18:7527-7534.

29. McCowan LM, North RA, Kho EM, Black MA, Chan EH, Dekker GA, Poston L, Taylor RS, Roberts CT: Paternal contribution to small for gestational age babies: a multicenter prospective study. Obesity (Silver Spring) 2011, 19:1035-1039.

30. Figueroa-Colon R, Arani RB, Goran MI, Weinsier RL: Paternal body fat is a longitudinal predictor of changes in body fat in premenarcheal girls. Am J Clin Nutr 2000, 71:829-834.

31. Kaati G, Bygren LO, Edvinsson S: Cardiovascular and diabetes mortality determined by nutrition during parents' and grandparents' slow growth period. Eur J Hum Genet 2002, 10:682-688.

32. Pembrey ME, Bygren LO, Kaati G, Edvinsson S, Northstone K, Sjostrom M, Golding J: Sex-specific, male-line transgenerational responses in humans. Eur J Hum Genet 2006, 14:159-166.

33. Pembrey ME: Male-line transgenerational responses in humans. Hum Fertil (Camb) 2010, 13:268-271.

34. McDonald SD, Han Z, Mulla S, Beyene J: Overweight and obesity in mothers and risk of preterm birth and low birth weight infants: systematic review and meta-analyses. BMJ 2010, 341:C3428.

35. Stothard KJ, Tennant PW, Bell R, Rankin J: Maternal overweight and obesity and the risk of congenital anomalies: a systematic review and meta-analysis. JAMA 2009, 301:636-650.

36. Wu Q, Suzuki M: Parental obesity and overweight affect the body-fat accumulation in the offspring: the possible effect of a high-fat diet through epigenetic inheritance. Obes Rev 2006, 7:201-208.

37. Carone BR, Fauquier L, Habib N, Shea JM, Hart CE, Li R, Bock C, Li C, Gu H, Zamore PD, Meissner A, Weng Z, Hofmann HA, Friedman N, Rando OJ: Paternally induced transgenerational environmental reprogramming of metabolic gene expression in mammals. Cell 2010, 143:1084-1096.

38. Braunschweig M, Jagannathan $V$, Gutzwiller A, Bee G: Investigations on transgenerational epigenetic response down the male line in $\mathrm{F} 2$ pigs. PLoS One 2012, 7:e30583.

39. Stein AD, Pierik FH, Verrips GH, Susser ES, Lumey LH: Maternal exposure to the Dutch famine before conception and during pregnancy: quality of life and depressive symptoms in adult offspring. Epidemiology 2009, 20:909-915.

40. Waterland RA, Kellermayer R, Laritsky E, Rayco-Solon P, Harris RA, Travisano M, Zhang W, Torskaya MS, Zhang J, Shen L, Manary MJ, Prentice AM: Season of conception in rural gambia affects DNA methylation at putative human metastable epialleles. PLoS Genet 2010, 6: e1001252.

41. Loomba R, Hwang SJ, O'Donnell CJ, Ellison RC, Vasan RS, D'Agostino RB Sr, Liang TJ, Fox CS: Parental obesity and offspring serum alanine and aspartate aminotransferase levels: the Framingham heart study. Gastroenterology 2008, 134:953-959.

42. Ng SF, Lin RC, Laybutt DR, Barres R, Owens JA, Morris MJ: Chronic high-fat diet in fathers programs beta-cell dysfunction in female rat offspring. Nature 2010, 467:963-966.

43. Dunn GA, Bale TL: Maternal high-fat diet promotes body length increases and insulin insensitivity in second-generation mice. Endocrinology 2009, 150:4999-5009.
44. Frystyk J, Vestbo E, Skjaerbaek C, Mogensen CE, Orskov H: Free insulin-like growth factors in human obesity. Metabolism 1995, 44:37-44.

45. Du Plessis SS, Cabler S, McAlister DA, Sabanegh E, Agarwal A: The effect of obesity on sperm disorders and male infertility. Nat Rev Urol 2010, 7:153-161.

46. Hoyo C, Fortner K, Murtha AP, Schildkraut JM, Soubry A, DemarkWahnefried W, Jirtle RL, Kurtzberg J, Forman MR, Overcash F, Huang Z, Murphy SK: Association of cord blood methylation fractions at imprinted insulin-like growth factor 2 (IGF2), plasma IGF2, and birth weight. Cancer Causes Control 2012, 23:635-645.

47. Murrell A, Ito Y, Verde G, Huddleston J, Woodfine K, Silengo MC, Spreafico F, Perotti D, De Crescenzo A, Sparago A, Cerrato F, Riccio A: Distinct methylation changes at the IGF2-H19 locus in congenital growth disorders and cancer. PLoS One 2008, 3:e1849.

48. Cruz-Correa M, Cui H, Giardiello FM, Powe NR, Hylind L, Robinson A, Hutcheon DF, Kafonek DR, Brandenburg S, Wu Y, He X, Feinberg AP: Loss of imprinting of insulin growth factor II gene: a potential heritable biomarker for colon neoplasia predisposition. Gastroenterology 2004 126:964-970

49. Cui H, Cruz-Correa M, Giardiello FM, Hutcheon DF, Kafonek DR, Brandenburg S, Wu Y, He X, Powe NR, Feinberg AP: Loss of IGF2 imprinting: a potential marker of colorectal cancer risk. Science 2003, 299:1753-1755.

50. Feinberg AP, Ohlsson R, Henikoff S: The epigenetic progenitor origin of human cancer. Nat Rev Genet 2006, 7:21-33.

51. Jirtle RL, Skinner MK: Environmental epigenomics and disease susceptibility. Nat Rev Genet 2007, 8:253-262.

52. Katari S, Turan N, Bibikova M, Erinle O, Chalian R, Foster M, Gaughan JP, Coutifaris C, Sapienza C: DNA methylation and gene expression differences in children conceived in vitro or in vivo. Hum Mol Genet 2009, 18:3769-3778.

53. Soubry A, Murphy SK, Huang Z, Murtha A, Schildkraut JM, Jirtle RL, Wang F, Kurtzberg J, Demark-Wahnefried W, Forman MR, Hoyo C: The effects of depression and use of antidepressive medicines during pregnancy on the methylation status of the IGF2 imprinted control regions in the offspring. Clin Epigenetics 2011, 3:2.

54. Barres R, Zierath JR: DNA methylation in metabolic disorders. Am J Clin Nutr 2011, 93:897S-900.

55. Pathak S, D'Souza R, Ankolkar M, Gaonkar R, Balasinor NH: Potential role of estrogen in regulation of the insulin-like growth factor2-H19 locus in the rat testis. Mol Cell Endocrinol 2010, 314:110-117.

56. Gemma C, Sookoian S, Alvarinas J, Garcia SI, Quintana L, Kanevsky D, Gonzalez CD, Pirola CJ: Maternal pregestational BMI is associated with methylation of the PPARGC1A promoter in newborns. Obesity (Silver Spring) 2009, 17:1032-1039.

57. Gicquel C, Rossignol S, Cabrol S, Houang M, Steunou V, Barbu V, Danton F, Thibaud N, Le Merrer M, Burglen L, Bertrand AM, Netchine I, Le Bouc Y: Epimutation of the telomeric imprinting center region on chromosome 11 p15 in Silver-Russell syndrome. Nat Genet 2005, 37:1003-1007.

58. Steenman MJ, Rainier S, Dobry CJ, Grundy P, Horon IL, Feinberg AP: Loss of imprinting of IGF2 is linked to reduced expression and abnormal methylation of H19 in Wilms' tumour. Nat Genet 1994, 7:433-439.

59. Honda S, Arai Y, Haruta M, Sasaki F, Ohira M, Yamaoka H, Horie H, Nakagawara A, Hiyama E, Todo S, Kaneko Y: Loss of imprinting of IGF2 correlates with hypermethylation of the $\mathrm{H} 19$ differentially methylated region in hepatoblastoma. $\mathrm{Br} J$ Cancer 2008, 99:1891-1899.

\section{Pre-publication history}

The pre-publication history for this paper can be accessed here: http://www.biomedcentral.com/1741-7015/11/29/prepub

doi:10.1186/1741-7015-11-29

Cite this article as: Soubry et al:: Paternal obesity is associated with IGF2 hypomethylation in newborns: results from a Newborn Epigenetics Study (NEST) cohort. BMC Medicine 2013 11:29. 